\section{IMPLEMENTATION OF A STRUCTURED EDUCATIONAL PATHWAY UTILISING FINAL YEAR STUDENT NURSES DURING A PANDEMIC}

Clare Paul, Hannah Fletcher. Great Ormond Street Hospital

10.1136/archdischild-2020-gosh.4

Rationale As the significant impact of COVID-19 became apparent, the government and Health Education England (HEE) recognised the vital role students would play throughout the pandemic. Students within 6 months of qualification elected to step into the role of band 4 Aspirant Nurses (ANs), working in a non-supernumerary capacity.

Skill gaps, with the potential to impact the AN's ability to work with the required level of autonomy, were identified upon induction. The ANs were supported by the Graduate and Undergraduate teams who collaborated to create a structured educational pathway. The outcome anticipated was to improve retention, decrease supernumerary time and shorten upskilling of newly registered competencies.

Description Preceptorship was introduced, acknowledging the transition from Student to Registered Practitioner and focusing on the required level of autonomy. A mandatory educational pathway included three study days combined with 570 hours of in-situ clinical teaching. These covered an induction to their role, oral and intravenous medication competencies, communication strategies, wellbeing and clinical simulation.

Results All ANs (100\%) who completed the programme reported that they found the teaching had a positive impact on their practice and that they would implement it within their role.

It is anticipated that the ANs transition to Registered Nurse will be accelerated, reflected in their shorter competency deadlines. Supernumerary time reduced by $50 \%$ for those entering new areas of practice whilst those remaining in the same area in which they completed student placement required no formal supernumerary time.

The effectiveness of this new role and education strategy will continue to be studied and reported as these ANs qualify and are compared with previous newly qualified cohorts. Whilst the circumstances which led to this teaching program may not be repeated, lessons learnt still have the potential to shape future transition.

\section{SYSTEM CHANGES IN RESPONSE TO THE COVID-19 PANDEMIC FOR ADOLESCENT MENTAL HEALTH CRISIS ADMISSIONS IN NORTH/CENTRAL LONDON}

Jon Goldin, Lee Hudson. Great Ormond Street Hospital and University College London

10.1136/archdischild-2020-gosh.5

As part of COVID 19 surge planning in March 2020 GOSH agreed to take paediatric patients from all the acute services in North Central London (UCLH, Royal Free, Whittington, Barnet and North Middlesex Hospitals). The plan was to close paediatric wards to make space for Adult Patients. Major systemic changes took place in less than 3 weeks with excellent collaborative work between all relevant stakeholders. An acute mental health inpatient ward was set up and was staffed by child and adolescent psychiatrists, mental health nurses and paediatricians/paediatric nurses. This presentation will describe the process of setting up this pathway and the patients that were referred. We shall explore aspects that went well as well as challenges we faced and areas of difficulty. The impact of the pandemic on mental health presentations will be considered, particularly with respect to self-harm, trauma, family environment, safeguarding, eating disorders and neurodevelopmental presentations. Clinical examples will be given. We shall also consider the impact of the pandemic on the mental health of staff. Outcomes of the pathway will be reflected upon as well as plans for the future and preparations for a possible 'second wave'.

\section{REDUCING PRE-ANALYTICAL ERRORS IN LABORATORY SAMPLES: TRUST-WIDE QUALITY IMPROVEMENT IN PAEDIATRIC HOSPITAL}

Malti Nakrani, Wisdom Musabaike, Christine Morris, Nuwanthi Yapa Mahathanthila. Great Ormond Street Hospital

\subsection{6/archdischild-2020-gosh.6}

Background Through manually recorded data, laboratory identified 4900 patient samples rejected in 2017 due to pre-analytical errors (PAE). This impacts patient safety and experience. QI project was started with aim to develop organization's capabilities in reducing PAEs.

Method A Steering committee was formed with stakeholders across the hospital. Using QI methodology drivers were identified. Working groups were formed. Participation was continuously reviewed to match the project at different stages. To engage frontline staff surveys, observations, focus groups were utilized.

The project investigated reasons for sample rejection: to understand the causes and ways to avoid them. Statistical Process Control charts for Outcome and Process measures were set and monitored as interventions were introduced through Plan-Do-Study-Act cycles. Key focus areas included: clotted samples, incorrect sample volumes, unnecessary stool testing and Blood Culture (BC) transport time. Continuous work with the Trust EPR system to improve rejection data quality was also key. Ethical approval was not required.

Results Multi-disciplinary engagement proved to be essential to this multi-factorial complex issue. Complexities in improving data accuracy provided significant learning, especially in terms of preparation for Electronic Patient Record system launch.

The most notable achievement being the reduction of $\mathrm{BC}$ transport time (collection-to-receipt in lab) from 265 to 116 minutes. The aim was to be under 120 minutes.

Clotted samples was one of the highest rejection reasons. Pre-intervention average was at 39 samples/week. This was reduced to 32 . Even though the interventions were successful, sustainability was difficult. The remainder of the improvements are supported through high quality data and an operational structure in place.

Discussion The project provided significant learning which is relevant and replicable for other paediatric/adult settings. Sustainability of education needs to be further explored.

Conclusion A hospital-wide approach is necessary to reduce PAE sample rejections where multiple complex factors lead to rejections. 